\title{
Reference value for the six-minute peg board and ring test. A cross sectional study
}

\author{
Manjiri Suhas Kulkarni, ${ }^{1}$ Gopala Krishna Alaparthi, ${ }^{2}$ Shyam Krishnan, ${ }^{1}$ Anand Ramakrishna, ${ }^{3}$ Vishak Acharya ${ }^{3}$ \\ ${ }^{1}$ Department of Physiotherapy, Kasturba Medical College, Manipal Academy of Higher Education, Mangalore, India \\ ${ }^{2}$ Department of Physiotherapy, College of Health Sciences, University of Sharjah, United Arab Emirates \\ ${ }^{3}$ Department of Pulmonary Medicine, Kasturba Medical College, Manipal Academy of Higher Education, Mangalore, India
}

Introduction: In our daily life, arm activities, whether supported or unsupported play a major role. Both simple and complex activities require the muscles, namely trapezius, pectoralis minor, scalene, and intercostals, to participate in arm positioning. These muscles also enact as the accessory respiratory muscles. Therefore, arm elevation increases the load on these muscles and they fail to perform dual activities, resulting in arm fatigue and a feeling of dyspnoea in healthy individuals as well as in chronic obstructive pulmonary disease patients. Various upper limb exercise tests were designed to measure this impairment, one of them being the six-minute peg board and ring test. The aim of the study is to derive a reference value for the six-minute peg board and ring test among healthy Indian population (Mangalore) from the age of 20-70 years of either gender. Also, to find a correlation among the number of rings and body mass index, arm length, arm and forearm circumference, the strength of shoulder and elbow flexors-extensors, grip strength of both sides and level of physical activity.

Methods: Participants performed two tests, thirty minutes apart. They were asked to load as many rings as possible in 6 minutes. Arm length, arm and forearm circumference were measured with a measuring tape. Shoulder and elbow flexors-extensors were assessed using a handheld push-pull dynamometer. Grip strength was measured with the Jamar hand-held dynamometer. Level of physical activity was assessed using International Physical Activity Questionnairelong form.

Results: The samples consisted of 450 healthy individuals between the age of 20-70 years. Reference values for each age group for both genders were reported. We found that age was correlated with the six-minute peg board and ring test score $(p<0.05)$. We also found a correlation between the strength variables and the test results $(p=0.001)$. However, no correlation was found between the arm length, arm and forearm circumference and the level of physical activity with the number of rings.

Conclusion: In this study, we derived a reference value for the six-minute peg board and ring test. There was a correlation among age, strength variables and the number of rings.

Key words: Upper extremity exercise test; six-minute peg board and ring test; exercise tolerance.

Correspondence: Dr. Gopala Krishna Alaparthi, Assistant Professor, Department of Physiotherapy, University of Sharjah, United Arab Emirates. E-mail: gopalalaparthi@gmail.com

Contributions: All authors contributed extensively in completion of this present study. All authors discussed the results and its implication, and gave their valuable inputs on the manuscript at all stages. All authors read and approved this manuscript.

Conflict of interest: The authors declare no conflict of interest.

Funding: This study did not receive any specific grant from any funding agency.

Availability of data and materials: The data supporting our findings are available from the corresponding authors upon reasonable request.

Ethics approval and consent to participate: Ethical clearance was obtained from the Institutional Ethics Committee of Kasturba Medical College, Manipal Academy of Higher Education, Mangalore, India, prior to the commencement of the study. Informed consent was obtained from every participant.

Consent for publication: Not applicable. 


\section{Introduction}

Arm activities, whether supported or unsupported, are commonly used for all the daily activities. They extensively contribute in performing both simple as well as complex everyday tasks, such as brushing, shaving, combing their hair, grooming activities, doing the dishes, or putting groceries on shelves, during which the muscles, namely - trapezius, pectoralis minor, scalene, and intercostals participate in arm positioning [1,2].

In healthy individuals and in patients with chronic obstructive pulmonary disease (COPD), a change in the respiratory muscle recruitment pattern is observed with the unsupported arm elevation, followed by an increase in the metabolic demand [2-10], the latter being more noticeable in patients with airflow obstruction. This can be explained by a decrease in efficiency of the respiratory mechanics, due to a dual activity of the shoulder girdle - which is to maintain the unsupported arm elevation and also participate in the process of respiration. This results in a shift of the respiratory workload on the diaphragm which is already mechanically disadvantaged in COPD [7]. Also, as the muscles that move the arms and give stability to the trunk are attached to the rib cage, it leads to an increase in the chest wall impedance, which further restricts the ability to increase the tidal volume during arm activities $[4,5,11]$.

As there is a reduction in the strength of the diaphragm muscle, patients may be unable to successfully shift this workload on the accessory muscles, leading to dyspnoea [7]. Therefore, activation of these muscle groups to perform two functions together, that is support the arm in elevation and also act as the accessory muscle of respiration, makes it difficult to perform upper limb activities asymptomatically. Numerous studies state that arm positioning limits the respiratory mechanics which is observed in the breathing pattern and increased thoraco-abdominal asynchrony during unsupported arm elevation $[9,12,13]$.

Over the years to quantify the impairment of unsupported upper limb function, various tests like the unsupported incremental upper limb exercise test (UULEX) [14], supported upper limb exercise test (SULEX) [14], grocery shelving test (GST) [15] and six-minute pegboard and ring test (6PBRT) [16] have been developed. The 6PBRT an easy and reasonable test that evaluates both arm function and endurance. It is time-bound (six minutes), and is validated and reproducible for healthy adults [17] as well as for individuals with COPD [16]. This test evaluates arm endurance by asking the subjects to move as many rings as possible from two lower pegs to two upper pegs in a six-minute time frame [16].

A recent study was conducted among the healthy Brazilian population to find the reference values for the 6PBRT. The results exhibited a correlation between the final 6PBRT value and age, indicating that individuals of the younger age could move more rings as compared to the older group. A weak correlation was also noted between the test results and the level of physical activity [1].

The normative value and reference range for the 6PBRT are currently unavailable for various ethnic background, including the Indian population. Hence, this study has been carried out to find out the reference value for 6PBRT.

\section{Methods}

\section{Study population and protocol}

We conducted a prospective cross-sectional study with the stratified sampling of healthy Indian (Mangalore) adults, recruited from two tertiary care hospitals. The aim of this study was to find out the reference values for the six-minute peg board and ring test, along with finding a correlation between the score of the test and BMI, arm length, arm and forearm circumference, shoulder and elbow flexors-extensors strength, grip strength and the level of physical activity. People accompanying the patients to these hospitals were considered, from the year 2018 to 2020 .

The inclusion criteria were: healthy adult between the age of 20 to 70 years, of either gender, having a body mass index (BMI) between $18.5 \mathrm{~kg} / \mathrm{m}^{2}$ to $40 \mathrm{~kg} / \mathrm{m}^{2}$.

The exclusion criteria were: any recent shoulder or thoracic surgery, any history of symptomatic heart or pulmonary diseases, and any musculoskeletal or neuromuscular or metabolic diseases and spinal deformities like kyphoscoliosis which would affect the performance of the protocol.

Materials required were: a chair, a peg board, 20 rings (14 g each), measuring tape, sphygmomanometer, pulse oximeter, stopwatch, stadiometer, weighing scale, stethoscope.

\section{Initial evaluation}

Demographic data was collected prior to the commencement of the test session. Height and weight were measured to calculate the BMI of individuals.

\section{Arm length, arm and forearm circumference of both dominant and non-dominant side}

These variables were measured using a measuring tape. Arm length was measured from the acromion process to the lateral border of the radial styloid process, with the arm in the anatomical position [17]. Arm circumference was measured midway between the acromion and olecranon process. Forearm circumference was measured near the olecranon, at its largest diameter, with the arm relaxed at the side of the body [18].

\section{Arm strength}

Shoulder and elbow flexor-extensor strength was measured with a hand-held push-pull dynamometer. Individuals were asked to contract isometrically with the dynamometer placed at different anatomical locations for a particular muscle group assessment. For shoulder flexors - shoulder joint was positioned in 90 degrees flexion, with the dynamometer placed at the distal end of humerus, about 5 centimetres above the elbow joint, whereas for shoulder extensor strength, upper extremity was placed at the side of the body in neutral position with the dynamometer placed posteriorly about 5 centimetres above the elbow joint [19]. For elbow flexorsshoulder joint was in the neutral position, elbow joint flexed at 90 degrees and in supination, the dynamometer was placed just proximal to the radial styloid process, with the anterior aspect of shoulder joint stabilized. In case of elbow extensor, shoulder joint was again in the neutral position, elbow joint flexed at 90 degrees and neutral, the dynamometer was placed proximal to the radial styloid process, with the anterior aspect of shoulder joint stabilised [20].

\section{Grip strength}

Jamar hand-held dynamometer was used. The subject was made to sit on a chair, back supported and arm rested on an armrest. The shoulder was adducted, elbow 90 degrees flexed, and forearm and wrist in mid-prone position. The subject was made to hold the dynamometer with the dominant hand first. The subject was asked to squeeze the dynamometer as much as they could. Three readings were taken with one minute rest among them. The best one of the 3 readings was considered. The therapist stood in front of the participant to read the amount of force (kg's). The same procedure was repeated on the other hand [21].

\section{Assessment of Physical Activity level}

International Physical Activity Questionnaire - Long form. This scale assessed the physical activity across a comprehensive 
set of 4 domains - leisure time physical activity, domestic and gardening (yard) activities, work-related physical activity and transport-related physical activity. Computation of the total scores required the summation of MET, duration (in minutes) and frequency (in days) for all the activities in all the domains. The subjects based on the values were categorized under low, moderate or high level of physical activity [22].

\section{Six-minute pegboard and ring test}

The test comprised of a peg-board with two sets of pegs attached, one at the level of the participant's shoulder (upper peg) and the other placed 20 centimetres below it (lower peg). These pegs were fixed at a distance which was equal to the shoulder width. Ten light-weight rings (approx. $14 \mathrm{~g}$ each) were placed on both the lower pegs, following which, the participant was asked to move a single ring at a time with both hands simultaneously from the lower peg to the upper. After shifting all the twenty rings, they were asked to move them back to the lower peg. Instructions were given to load as many rings as possible in the time duration of six minutes. Vitals were recorded before and after the test. At the end, the number of rings moved were documented. Standard words of encouragement were given during the performance of the test. A trial was done prior to the commencement of the protocol in order to acquaint the participant with the test procedure (Figure 1) [1].

\section{Statistical analysis}

Statistical analysis was performed using IBM SPSS statistics for Windows, ver. 25.0 (IBM Corp., Armonk, NY, USA). The average number of rings for each age group was described as mean and standard deviation at $95 \% \mathrm{CI}$, with the lower and upper limit considered as lower and upper range of values respectively. The correlation among the performance of the 6PBRT, age, BMI, arm length, arm and forearm circumference, grip strength, arm strength for either side was assessed using Pearson's correlation test. The association between the level of physical activity and the 6PBRT score was assessed using ANOVA and Bonferroni's test. Significance between the pre and post values of the hemodynamic variables found was evaluated using the Wilcoxon's test. The association between the number of rings completed and the independent variables was tested by simple linear regression analysis, and stepwise linear regression was done to derive equations with the coefficient $\left(\mathrm{r}^{2}\right)$.

\section{Results}

A total of 450 healthy individuals were included with 90 healthy adults in each age groups of 20-29, 30-39, 40-49, 50-59 and 60-69. All the groups contained 45 males and 45 females. The mean number of rings completed by females and males are presented in Tables 1 and 2. A strong correlation was found between the pre and post-test hemodynamic values in each age group in either gender $(p<0.001)$.

Figures 2 and 3 show a strong negative correlation between the age and number of rings in both females and males. No statistically significant correlation was noted between the number of rings loaded and the arm length and the arm circumference of both the dominant and non-dominant side. However, a statistical significance was seen between forearm circumference in males and the number of rings, but with no correlation between these two variables (i.e., $r<0.3$ ), whereas the female group showed no significant correlation between them (Table 3 ).

Table 4 shows a moderate to a strong correlation between the number of rings loaded and the strength of shoulder flexors-extensors, elbow flexors-extensors and grip strength of both sides in all the age groups in females as well as males. As a significant correlation was seen in between the strength variables, age and the number of rings, a stepwise multiple linear regression analysis was done. For females- age and the grip strength of right hand (gripr) were selected as the predictors of the 6PBRT values, and for malesage, shoulder extensor strength of left hand (shextl) and elbow extensors of the right hand (elbowextr) were selected.
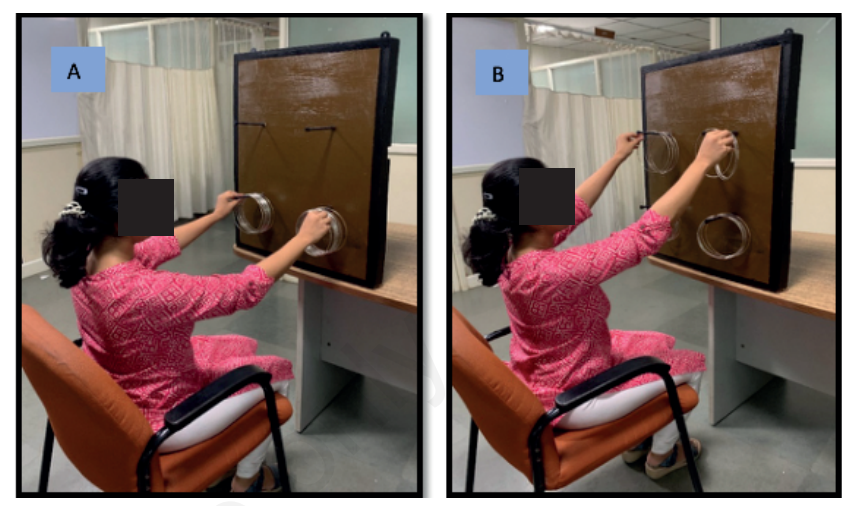

Figure 1. A) Participant starting to move rings from lower peg to upper peg. B) The same participant moving the ring from upper peg to lower peg.

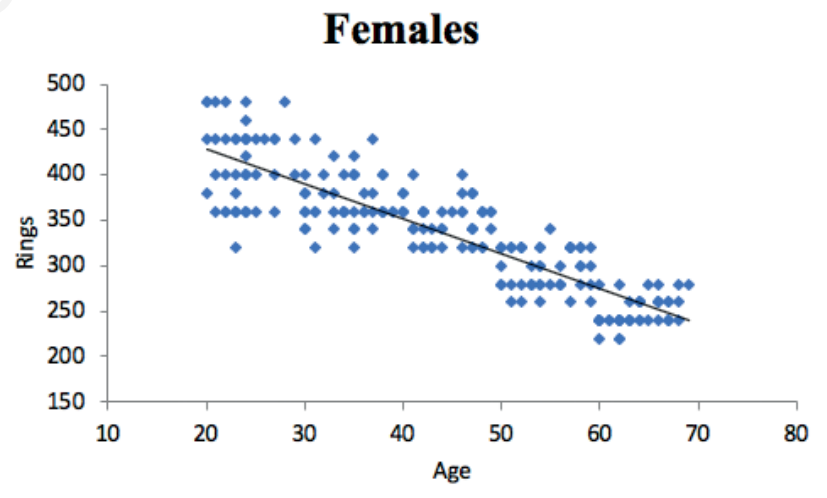

Figure 2. Relation between the number of rings and age in females.

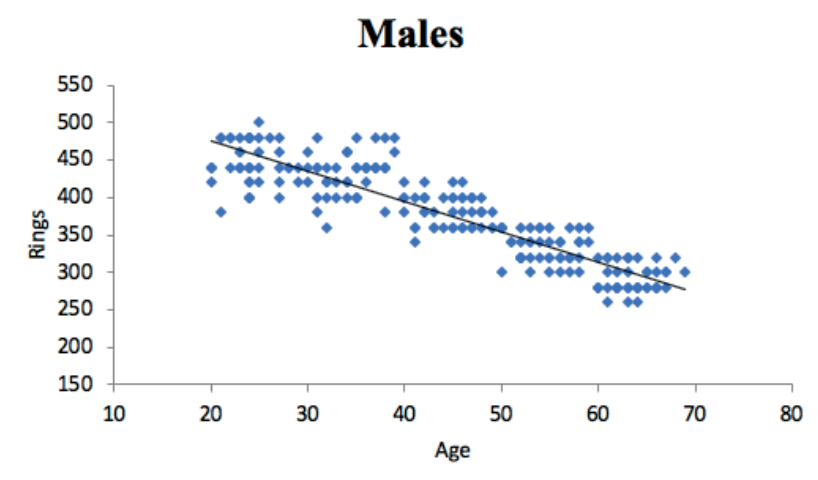

Figure 3. Relation between the number of rings and age in males. 
Equation 1 (for females): 6 PBRT $=572.338+(4.580 \times$ gripr $)+$ $(-5.619 \times$ age $), r^{2}=0.605$

Equation 2 (for males): 6 PBRT $=388+(16.561 \times$ shextl $)+$ $(20.4 \times$ elbowextr $)+(-3.69 \times$ age $), r^{2}=0.685$.

The results also depicted that there was no association between the number of rings and the level of physical activity, thus concluding that the number of rings completed in the 6PBRT in 6 minutes is independent of physical activity levels of an individual.

\section{Discussion}

The present study aimed to provide normative values for the 6PBRT in healthy individuals among the Mangalore population. A total of 450 individuals with equal representation from either gender were recruited and divided into five $(20-29,30-39,40-49,50-$ 59 and 60-69) groups of 90 subjects each.

The descriptive statistics for the number of rings loaded onto the pegs as per the standardized test protocol for either gender in each age group was estimated separately across all the age groups, and the general observation was that males performed better in terms of the number of rings loaded. This variation might be reflected due to various factors like difference in muscle mass, gender-specific muscle fibres which depend on hormones - like testosterone which is responsible for an increase in the muscle mass. Also, muscle power, which is determined by the fast-twitch fibres (type IIa), is more in number in males as compared to females. All these factors together favour men to perform physically better than women [23]. Nevertheless, a study conducted on the Brazilian population to find the normative value for 6PBRT found contradictory results with no such association between the number of rings and gender in age-matched individuals [1].

The number of rings steadily decreased as the age group progressed. Subjects in the youngest age group of 20-29 years were able to move a greater number of rings as compared to the oldest group of 60-69 years. For the older age group, factors that have been attributed to poorer performance in the 6PBRT are changes in muscle mass, a marked reduction in the muscle strength, endurance and efficiency of performing fine motor activities [24,25]. Another possible explanation for the same can be a decrease in the ability perceive the upper limb position while performing the test, which may be further associated with a decline in proprioceptive abilities and memory, even in the healthy individuals [26]. A previous normative study in Brazilian population also showed a similar trend in terms of the number of rings moved during the 6PBRT [1].

Pre and post-test values of the hemodynamic variables like heart rate, respiratory rate, systolic and diastolic BP, and saturation along with dyspnoea and fatigue (upper limb) with the help of Borg's scale were recorded to find out the effects of 6PBRT on these variables. It was concluded that there was a small but significant difference between the pre and post values of each of the above-mentioned variables. This can be explained by the fact that exercising muscles lead to an increase in the metabolic demand, which is met through various changes in the respiratory and circulatory system. The sympathetic nervous system plays a major part in increasing heart rate, respiratory rate and blood pressure- systolic as well as diastolic during exercise [27].Our findings are similar to a study done by Ohara et al., in which the pre-post vitals after performing 6PBRT was checked, and found to have significant correlation [28].

We hypothesized that variables such as weight, height, BMI, arm length, arm and forearm circumference, shoulder, elbow flex-
Table 1. Number of rings loaded by females.

\begin{tabular}{lccc} 
Age group & n & $\begin{array}{c}\text { Mean } \pm \text { standard } \\
\text { deviation }\end{array}$ & $\begin{array}{c}\text { 95\% Confidence } \\
\text { interval }\end{array}$ \\
\hline $20-29$ & 45 & $412.44 \pm 43.33$ & $399.42-425.46$ \\
$30-39$ & 45 & $378.22 \pm 53.90$ & $362.02-394.41$ \\
\hline $40-49$ & 45 & $350.22 \pm 22.81$ & $343.36-357.07$ \\
$50-59$ & 45 & $296.88 \pm 22.13$ & $290.88-303.53$ \\
\hline $60-69$ & 45 & $248.88 \pm 16.26$ & $244.00-253.77$ \\
\hline
\end{tabular}

Table 2. Number of rings loaded by males.

\begin{tabular}{lccc} 
Age group & $\mathbf{n}$ & $\begin{array}{c}\text { Mean } \pm \text { standard } \\
\text { deviation }\end{array}$ & $\begin{array}{c}\text { 95\% Confidence } \\
\text { interval }\end{array}$ \\
\hline $20-29$ & 45 & $447.11 \pm 28.65$ & $438.50-455.71$ \\
$30-39$ & 45 & $432.88 \pm 28.33$ & $424.37-441.40$ \\
\hline $40-49$ & 45 & $383.11 \pm 20.86$ & $376.84-389.38$ \\
$50-59$ & 45 & $333.33 \pm 20.44$ & $327.18-339.47$ \\
\hline $60-69$ & 45 & $291.55 \pm 18.33$ & $286.04-297.06$ \\
\hline
\end{tabular}

Table 3. Correlation among the number of rings loaded and the arm length, arm and forearm circumference in females and males.

\begin{tabular}{lcccc} 
Variables & & & Females & Males \\
Arm length & \multirow{2}{*}{ Right } & $\mathrm{r}$ & 0.108 & 0.050 \\
& & $\mathrm{p}$ & 0.107 & 0.451 \\
& Left & $\mathrm{r}$ & 0.106 & 0.053 \\
& & $\mathrm{p}$ & 0.112 & 0.430 \\
Arm circumference & \multirow{2}{*}{ Right } & $\mathrm{r}$ & -0.044 & 0.028 \\
& & $\mathrm{p}$ & 0.508 & 0.678 \\
& \multirow{2}{*}{ Left } & $\mathrm{r}$ & -0.044 & 0.028 \\
Forearm circumference & \multirow{2}{*}{ Right } & $\mathrm{p}$ & 0.508 & 0.678 \\
& & $\mathrm{r}$ & 0.045 & 0.211 \\
& \multirow{2}{*}{ Left } & $\mathrm{p}$ & 0.499 & $0.001^{*}$ \\
& & $\mathrm{r}$ & 0.047 & 0.211 \\
& & $\mathrm{p}$ & 0.487 & $0.001^{*}$ \\
\hline
\end{tabular}

${ }^{*} p<0.05$ : significant.

Table 4. Correlation between the number of rings loaded and the strength variables of the upper limb in females and males.

\begin{tabular}{llccc} 
Variables & & & Females & Males \\
Shoulder flexors & Right & r & 0.375 & 0.493 \\
& & $\mathrm{p}$ & $0.000^{*}$ & $0.000^{*}$ \\
& Left & $\mathrm{r}$ & 0.452 & 0.514 \\
& & $\mathrm{p}$ & $0.000^{*}$ & $0.000^{*}$ \\
Shoulder extensors & Right & $\mathrm{r}$ & 0.520 & 0.522 \\
& & $\mathrm{p}$ & $0.000^{*}$ & $0.000^{*}$ \\
& Left & $\mathrm{r}$ & 0.561 & 0.590 \\
& & $\mathrm{p}$ & $0.000^{*}$ & $0.000^{*}$ \\
\hline Elbow flexors & Right & $\mathrm{r}$ & 0.488 & 0.572 \\
& & $\mathrm{p}$ & $0.000^{*}$ & $0.000^{*}$ \\
& Left & $\mathrm{r}$ & 0.509 & 0.486 \\
& & $\mathrm{p}$ & $0.000^{*}$ & $0.000^{*}$ \\
Elbow extensors & Right & $\mathrm{r}$ & 0.475 & 0.686 \\
& & $\mathrm{p}$ & $0.000^{*}$ & $0.000^{*}$ \\
& Left & $\mathrm{r}$ & 0.480 & 0.461 \\
& & $\mathrm{p}$ & $0.000^{*}$ & $0.000^{*}$ \\
\hline Grip strength & Right & $\mathrm{r}$ & 0.650 & 0.513 \\
& & $\mathrm{p}$ & $0.000^{*}$ & $0.000^{*}$ \\
& Left & $\mathrm{r}$ & 0.622 & 0.453 \\
& & $\mathrm{p}$ & $0.000^{*}$ & $0.000^{*}$ \\
\hline
\end{tabular}

${ }^{*} p<0.05$ : significant. 
ors- extensors strength and grip strength would be the predictors of the number of rings completed in the test in 6 minutes. There was no significant correlation seen among height, weight and BMI in either gender with the number of rings which corroborates with the findings of Ohara et al. [28]. BMI does not interfere with the performance of the test, probably due to the fact that 6PBRT does not intend to test the exercise capacity or intolerance through full-body activities, rather the participants are seated on a chair throughout the test. So, we can conclude that whether an individual is categorized as normal or obese based on the BMI value, it has no effect on the performance of the test.

To our knowledge, this is the first study to correlate the arm length, arm circumference and forearm circumference with the number of rings completed in the 6PBRT among healthy Indian population. No statistically significant correlation was found among these variables. One of the possible reasons as to why arm length does not affect the performance of 6PBRT can be that, since the protocol does not state about placing the chair on which the participant sits at a fixed distance from the board, the chair can be moved, and the distance between the chair and the board can be adjusted to ensure the participants' reachability for both the lower and upper pegs. On the other hand, arm and forearm circumference do not necessarily indicate about the muscle mass and strength; even fat tissues contribute to the girth. Therefore, we can conclude that circumference has nothing to do with the strength of the upper limbs and so it would have no effect on the performance of the 6PBRT. Similarly, a study done by Lima et al. [1] on healthy Brazilian population to find a correlation between the arm and forearm circumference of the dominant hand, concluded that there was no significant correlation between the length, circumference and the 6PBRT ring score. It means that the performance of the test does not depend on short or long arms or having smaller or larger arm and forearm circumference, which does support our results.

The existing literature supports the fact that muscles around the shoulder and elbow joint play an important role as accessory muscles when the primary respiratory muscles fail to meet the ventilatory demand [29]. As arm activities participate in the majority of our daily activities, in healthy individuals as well as in COPD, the strength of these muscle groups is important, as they have to perform dual activities. If the strength is better, chances for subjects to be symptomatic while performing ADL's using their upper limbs reduces. So, in our study, we assessed the relationship between the muscle strength of the major muscle group of the upper extremity of the dominant and non-dominant side and the number of rings and it was found that there is a statistically significant correlation between the muscle strength and performance of the 6PBRT. However, this relationship was not observable in individual age groups of either gender. Janaudis-Ferreira et al. [30], in their study, evaluated the correlation between the shoulder and elbow flexors strength with the total score of rings loaded in the 6PBRT. Their study demonstrated a moderate to a strong correlation between the shoulder and elbow flexion strength with the total score of the test, which gave the inference that the muscle groups of these joints have an important relationship with the arm activities, thus supporting our results.

Grip strength of both the dominant and non-dominant side was also correlated with the 6PBRT score, demonstrating a significant correlation with the score in either gender, which contradicts the results of a study done by Ohara et al. [28]. The importance of grip strength in performing the test may be due to the fact the rings need to be held with both hands simultaneously and moved between the two levels of pegs. More the grip strength, better the ability to grab the rings, and hence will be able to load a greater number of rings in the given time, thus increasing the 6PBRT score.

Level of physical activity was assessed with the International Physical Activity Questionnaire - long form, and its association with the number of rings loaded was assessed. No association was seen between these two variables, probably because, the participant was asked to sit on a chair and perform this test. Therefore, the performance may not be influenced by a high or a low level of physical fitness. Also, the components of the questionnaire concentrated more on activities performed by the lower limbs - walking and running, which in turn make it difficult to categorize the level of activity performed by the upper limbs and then compare it with the 6PBRT scores [28]. Our findings differ from the Brazilian study in which they evaluated an association between the level of physical activity using the Human Activity Profile (HAP) questionnaire and the number of rings in the healthy population, and found a weak correlation between them [1].

Limitations of the present study were that we did not consider the time of testing as there could be confounding factors like medications taken for any medical conditions. We also did not consider the motivation levels and other psychological functions while the study was performed. Many samples in the older age group were lost due to comorbidities like frozen shoulder or hemiparetic limb as it would interfere with the performance of the test.

\section{Conclusions}

In conclusion, the present study was able to determine the reference values for the 6PBRT in healthy Indian population in the age of 20-70 years of either gender. There was a strong negative correlation seen between the age and number of rings, inferring that older the individual, lesser is the 6PBRT score. We also found a correlation between the strength variables of the upper limb and the number of rings exchanged. Physical activity levels, as well as BMI, had no correlation with the number of rings.

\section{List of abbreviation}

6PBRT: $\quad$ six-minute peg board and ring test;

BMI: body mass index;

ADL: $\quad$ activities of daily living;

COPD: $\quad$ chronic obstructive pulmonary disease;

MET: metabolic equivalent.

\section{References}

1. Lima VP, Almeida FD, Janaudis-Ferreira T, Carmona B, Ribeiro-Samora GA, Velloso M. Reference values for the six minute peg board and ring test in healthy individuals in Brazil. J Bras Pneumol 2018;44:190-4.

2. Couser JI Jr, Martinez FJ, Celli BR. Respiratory response and ventilatory muscle recruitment during arm elevation in normal subjects. Chest 1992;101:336-40.

3. Celli B, Criner G, Rassulo J. Ventilatory muscle recruitment during unsupported arm exercise in normal subjects. J Appl Physiol 1988;64:1936-41.

4. Baarends EM, Schols AMWJ, Slebos DJ, Mostert R, Janssen PP, Wouters EF. Metabolic and ventilatory response pattern to arm elevation in patients with COPD and healthy age-matched subjects. Eur Respir J 1995;8:1345-51.

5. Criner GJ, Celli BR. Effect of unsupported arm exercise on ventilatory muscle recruitment in patients with severe chronic airflow obstruction. Am Rev Respir Dis 1988;138:856-61.

6. Dodd DS, Brancatisano T, Engel LA. Chest wall mechanics during exercise in patients with severe chronic airflow obstruction. Am Rev Respir Dis 1984;129:33-8. 
7. Epstein SK, Celli BR, Williams J, Tarpy S, Roa J, Shannon T. Ventilatory response to arm elevation. Its determinants and use in patients with chronic obstructive pulmonary disease. Am J Respir Crit Care Med 1995;152:211-6.

8. Martinez FJ, Couser JI, Celli BR. Factors influencing ventilatory muscle recruitment in patients with chronic airflow obstruction. Am Rev Respir Dis 1990;142:276-82.

9. Martinez FJ, Couser JI, Celli BR. Respiratory response to arm elevation in patients with chronic airflow obstruction. Am Rev Respir Dis 1991;43:76-80.

10. Velloso M, Stella SG, Cendon S, Silva AC, Jardim JR. Metabolic and ventilatory parameters of four activities of daily living accomplished with arms in COPD patients. Chest 2003;123:1047-53.

11. Kathiresan G, Jeyaraman SK, Jaganathan, J. Effects of upper extremity exercise in people with COPD. J Thorac Dis 2010;2:223-36.

12. Celli BR, Rassulo J, Make BJ. Dyssynchronous breathing during arm but not leg exercise in patients with chronic airflow obstruction. N Engl J Med 1986;314:1485-90.

13. Dolmage TE, Maestro L, Avendano MA, Goldstein RS. The ventilatory response to arm elevation of patients with chronic obstructive pulmonary disease. Chest 1993;104:1097-100.

14. Takahashi T, Jenkins SC, Strauss GR, Watson CP, Lake FR. A new unsupported upper limb exercise test for patients with chronic obstructive pulmonary disease. J Cardiopulm Rehabil 2003;23:430-7.

15. Hill CJ, Denehy L, Holland AE, McDonald CF. Measurement of functional activity in chronic obstructive pulmonary disease: the grocery shelving task. J Cardiopulm Rehabil 2008;28:402-9.

16. Zhan S, Cerny FJ, Gibbons WJ, Mador MJ, Wu YM. Development of an unsupported arm exercise test in patients with chronic obstructive pulmonary disease. J Cardiopulm Rehabil 2006;26:180-7.

17. Eston R, Hawes M, Martin A, Reilly T. Human Body Composition. In: Eston R, Reilly T, editors. Kinanthropometry and exercise physiology laboratory manual, 3rd ed. Oxon: Routledge; 2009. p. 46.

18. Sagun G, Oguz A, Karagoz E, Filizer A, Tamer G, Mesci B. Application of alternative anthropometric measurements to predict metabolic syndrome. Clinics 2014;69:347-53.

19. Rawat P, Eapen C, Seema KP. Effect of rotator cuff strength- ening as an adjunct to standard care in subjects with adhesive capsulitis: A randomized controlled trial. J Hand Ther 2017;30:235-41.

20. Andrews AW, Thomas MW, Bohannon RW. Normative values for isometric muscle force measurements obtained with hand held dynamometers. Phys Ther 1996;76:248-59.

21. Massy-Westropp N, Gill T, Taylor A, Bohannon R, Hill C. Hand grip strength: age and gender stratified normative data in a population-based study. BMC Res Notes 2011;4:127.

22. International Physical Activity Questionnaire (IPAQ). Guidelines for data processing and analysis of international physical activity questionnaire (IPAQ) - short and long forms. Available from: https://sites.google.com/site/theipaq/home

23. Mohan S, Bagavad Geetha M, Padmavathi R. Study of gender variation in muscle function among young adults. Natl J Physiol, Pharm Pharmacol 2017;7:793-6.

24. Hoogendam YY, van der Lijn F, Vernooij MW, Hofman A, Niessen WJ, van der Lugt A, et. al. Older age relates to worsening of fine motor skills: a population-based study of middleaged and elderly persons. Front Aging Neurosci 2014;6:25965 .

25. Ingram LA, Butler AA, Walsh LD, Brodie MA, Lord SR, Gandevia SC. The upper limb Physiological Profile Assessment: Description, reliability, normative values and criterion validity. PloS One 2019;14:e0218553.

26. Adamo DE, Alexander NB, Brown SH. The influence of age and physical activity on upper limb proprioceptive ability. J Aging Phys Act 2009;17:272-93.

27. Thimmaraju L, Anandarao SB. Gender differences in cardiovascular response to upper limb isometric exercises. Int J Res Health Sci 2014;2:454-61.

28. Ohara DG, Melo CS, Reis IM, Jamami M. Functional capacity assessment of upper limbs in healthy young adult subjects. Fisioter Mov 2017;30:159-67.

29. Liu X, Li P, Wang Z, Lu Y, Li N, Xiao L, et al. Evaluation of isokinetic muscle strength of upper limb and the relationship with pulmonary function and respiratory muscle strength in stable COPD patients. Int J Chron Obstruct Pulmon Dis 2019;14:2027-36.

30. Janaudis-Ferreira T, Hill K, Goldstein RS, Wadell K, Brooks D. Relationship and responsiveness of three upper-limb tests in patients with chronic obstructive pulmonary disease. Physiother Can 2013;65:40-3.

Received for publication: 10 April 2020. Accepted for publication: 4 May 2020.

This work is licensed under a Creative Commons Attribution-NonCommercial 4.0 International License (CC BY-NC 4.0).

(C) Copyright: the Author(s), 2020

Licensee PAGEPress, Italy

Multidisciplinary Respiratory Medicine 2020; 15:670

doi:10.4081/mrm.2020.670 